\title{
Application of Trio Motion Controller in the Control System of Glass Engraving Machine
}

\author{
Hua-Yang Zhao ${ }^{1, a}$, Da-Peng Liü ${ }^{2, b}$ \\ ${ }^{1}$ Inner Mongolia University for the Nationalities, College of Mechanical Engineering, TongLiao, \\ 028000, China; \\ ${ }^{2}$ NO.724 Research Institute under CSIC, Nanjing Jiangsu 210003, China;
}

Keywords: Trio Motion controller, Openness, Control system, Glass engraving machine.

\begin{abstract}
In recent years, glass engraving products get the favor of consumers, demand of glass engraving products become higher and higher, the traditional glass engraving is mainly done manually, labor intensity is big, the work efficiency is low, in view of this situation, a kind of glass engraving machine based on Trio motion controller is designed in this paper. Hardware design and software design have been introduced. The system has high openness and high stability, and it has a certain practical significance for glass engraving industry.
\end{abstract}

\section{Introduction}

With social progress and people's living standards improving, carved glass products get more and more attention of consumers and glass engraving is to carve the characters and patterns or patterns on the glass, it is available as decorations, crafts and so on, it is beautiful and generous [1]. Consumer requirements are continuous improving, glass carving technology is also increasingly complex, precision increasingly is high [2]. The engraving process of the products of glass on the market is mainly by manual operation to complete, processing efficiency is low, quality cannot be guaranteed, the comprehensive cost is high [3]. In order to further expand the market of glass carving products, CNC engraving equipment has a broad prospect [4]. The glass engraving machine is mainly Italy company production in the market [5]. In order to change this situation, this paper describes the design of a CNC glass engraving machine based on Trio motion controller. The control system adopts the open numerical control system of "PC + motion controller" control mode, it can enhance the flexibility and adaptability of the system. It has a certain practical significance for the development of China's glass carving industry.

\section{The system hardware structure}

Introduction of Trio motion controller. TRIO digital motion controller is a kind of digital motion controller developed based on micro processing technology of high precision and high function by Trio motion technology Ltd. TRIO digital motion controller combines motion control and logic algorithm, it can easily achieve a variety of forms of exercise, such as linear interpolation, circular interpolation, helix interpolation, electronic gearing and electronic cam. TRIO digital motion controller has good expansibility and provides a variety of functional modules, it can configure according to the application requirements of on-demand portfolio and it improves the system openness and portability. There are many connections with the servo driver. Such as CANopen (Controller Area Networking)、DeviceNET、Profibus、SERCOS (Serial Realtime Communication System)、SLM (Speed Loop Module). And Trio motion controller programming is convenient, it uses trio basic programming language, which is similar to the Basic language, it is easy to learn and use.

Engraving machine mechanical structure design. Mechanical body of engraving machine adopts gantry structure, it has four degrees of freedom altogether, respectively they are $\mathrm{X}, \mathrm{Y}, \mathrm{Z}$ three mobile freedom and $\mathrm{C}$ a rotation degree of freedom. The spindle is a rotating axis driven by wheel speed. C axis can control the attitude angle of grinding wheel shaft. So the engraving machine is 
totally five drive shafts, the glass engraving is realized with the linkage of axis $\mathrm{X}, \mathrm{Y}, \mathrm{Z}$ and $\mathrm{C}$ axis. As shown in Figure 1, X, Y, Z axis connect directly to ball screw to drive by servo motor, $\mathrm{C}$ axis servo motor is connected with the speed reducer to drive. The engraving machine get the graphics settings of glass grinding through the high speed emery wheel rotating driven by spindle.

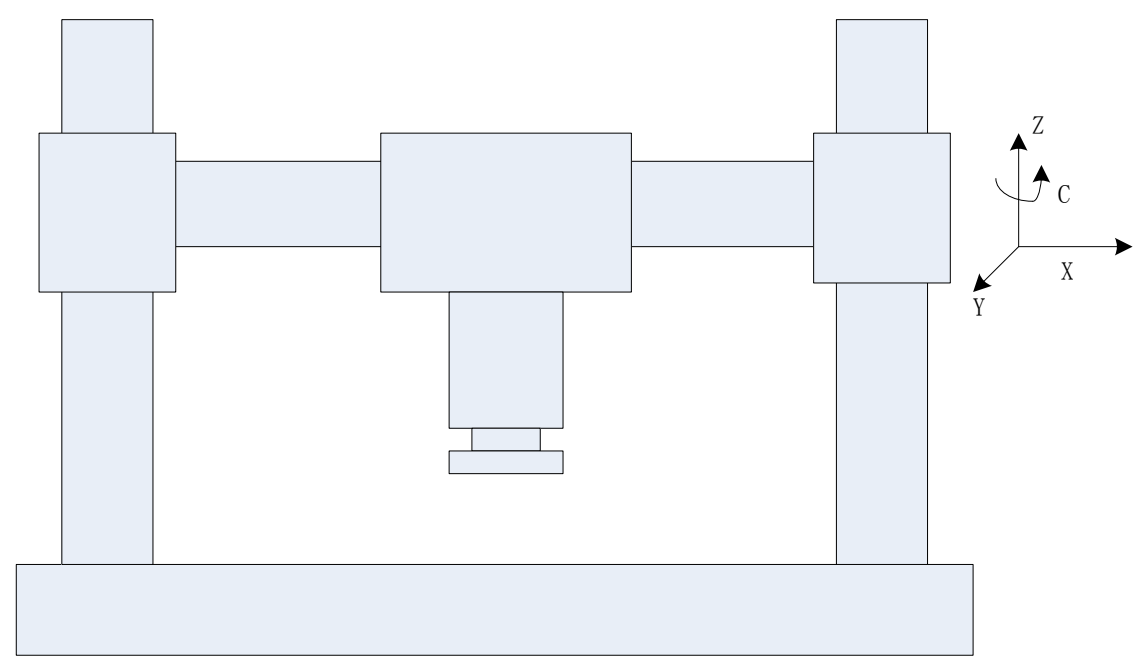

Figure1. Mechanical body of engraving machine

Engraving machine control system design. Engraving machine control system is shown in Figure 2, the system uses the modular design. The control system consists of PC machine, Trio motion controller, servo driver and servo motor. PC is the operation and management module, it is used for system operation and program management; Trio motion controller is control module and also it is the core of the whole control system, connection between the PC and Trio motion controller uses Ethernet, they can communicate directly. Trio motion controller receives signals from the PC machine, and controls each axis, the engraving machine makes the corresponding motion. Servo driver and servo motor and actuator are engraving machine body module, it completes the glass processing according to the instructions of the system. Engraving machine modular design is shown in figure 3.

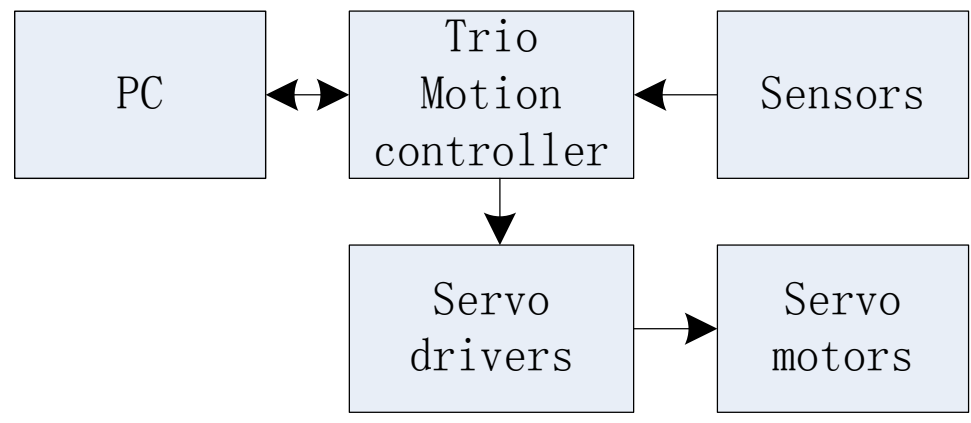

Figure 2. The hardware design 


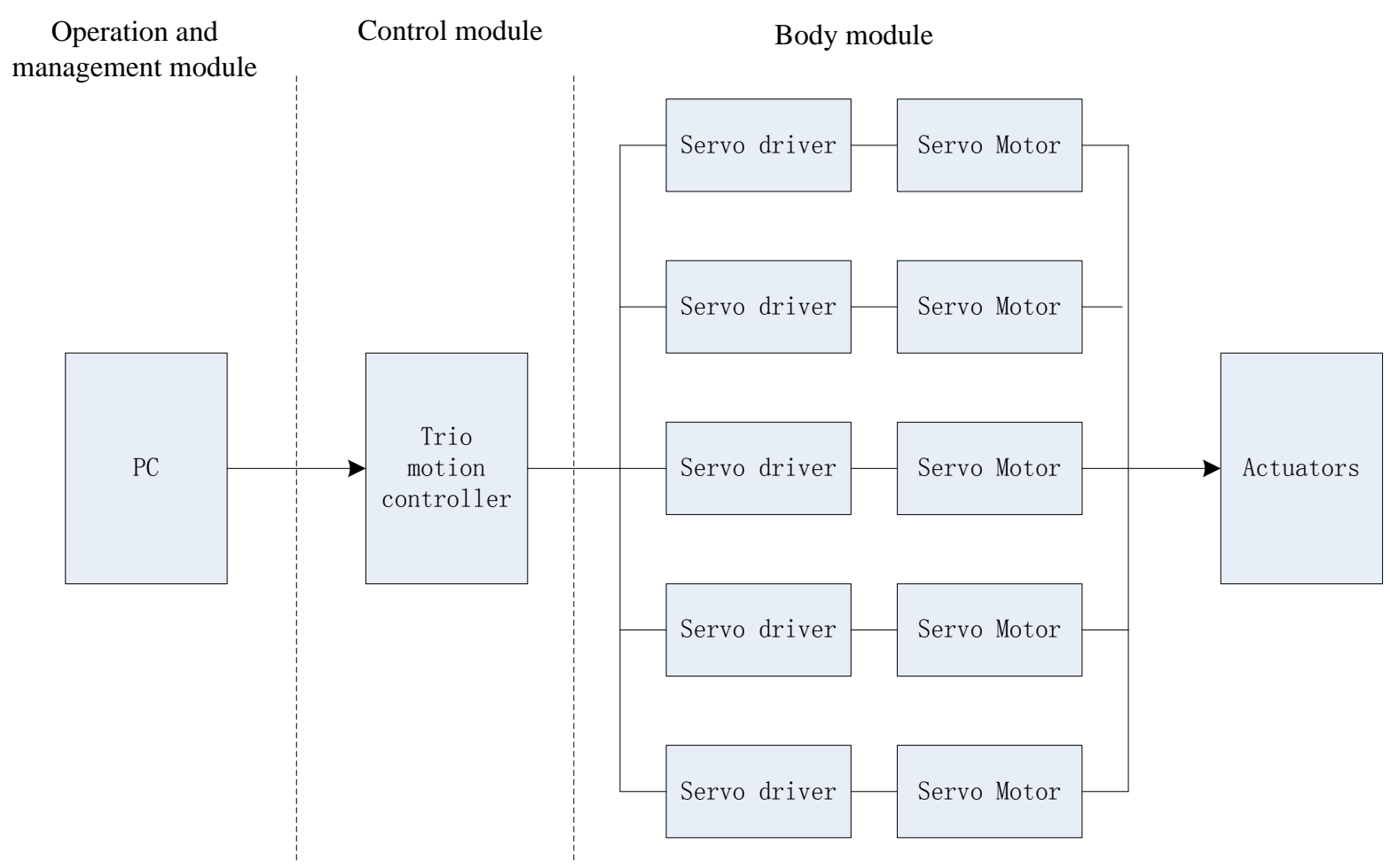

Figure3. The modular design of hardwar

\section{Software design}

Software structure design. Trio motion controller is the core of the engraving machine control system. As shown in Figure 4, the control system uses the form of upper and lower computer. The trio motion controller is running based on a PC, motion controller only has operating environment, there is no development environment, so it is necessary to use PC as the upper computer, motion controller as lower computer. Upper computer is mainly responsible for the management and operation, lower computer is used to perform motion program, programs are written separately between the upper computer and the lower computer.

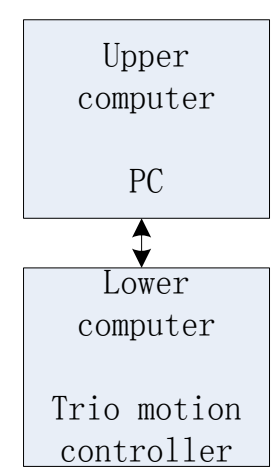

Figure4. Software structure

The upper computer program. As shown in Figure 5, the upper computer program is mainly used for parameter setting and display, the graphics transformation, fault diagnosis and real-time communication. Trio motion controller software Motion perfect can use VC++ or VB to develop second times. At the same time, CADtoMotion can directly do the transformation of $2 \mathrm{D}$ graphics of 
AutoCAD drawing for the program and download it to the trio motion controller, which greatly reduces workload of programming.

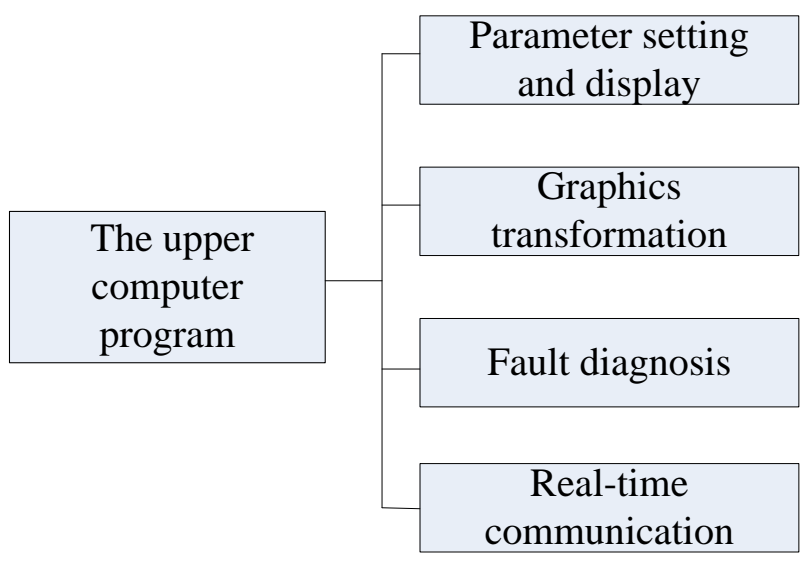

Figure5. The upper computer program

The lower computer program. As shown in Figure 6, the lower computer program is written on a computer using Motion perfect software, it can be on-line debugging. It consists of program module, interpolation module, program explaining module and input and output module. The interpolation module includes linear interpolation, circular interpolation, helix interpolation etc. User input data can be converted to processing procedures through program explaining module.

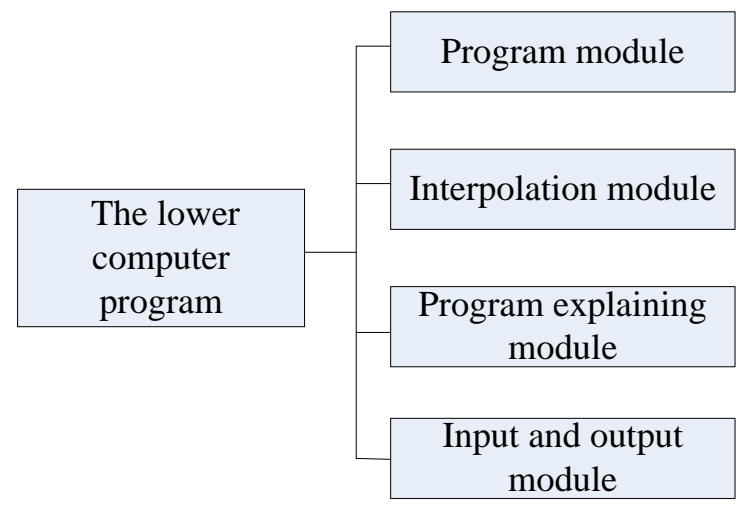

Figure6. The lower computer program

I/O design. The system sends and receives signal through the I/O interface controller, including the zero returning signal, position limit signal and indicating lights. The design of I/O system is shown in table 1.

Table1. System I/O design

\begin{tabular}{lllc}
\hline & Input & & Output \\
\hline IN0 & Zero returning signal 1 & OUT0 & Indicating light 1 \\
IN1 & Zero returning signal 2 & OUT1 & Indicating light 2 \\
IN2 & Zero returning signal 3 & OUT2 & Indicating light 3 \\
IN3 & Position limit signal 1 & OUT3 & Indicating light 4 \\
IN4 & Position limit signal 2 & OUT4 & Indicating light 5 \\
IN5 & Position limit signal 3 & OUT5 & Indicating light 6 \\
\hline
\end{tabular}

\section{Summary}

Aiming at the shortage of traditional glass carving process, a kind of CNC glass engraving machine based on Trio motion controller is designed. Open numerical control system based on trio motion controller has high stability, good openness, fast running speed, and higher control precision. The 
system adopts double CPU structure, modular design makes software development easier, which helps to transform and upgrade the system. It will provide a new idea that motion controller is applied to the glass engraving machine for glass engraving, which has a certain practical significance.

\section{Acknowledgement}

This research was financially supported by the The Inner Mongolia Autonomous Region Castor industry engineering technology research center Open fund projectReferences (BMYJ 2015-04).

\section{Reference}

[1] Zhu Xun, Fu Jianzhong. CNC Glass Engraver Machine Center of synchronous control method and application [J]. Combination machine tools and automatic processing technology, 2003, (12): 85-86.

[2] Xiong Wenli. The application of emotional design of glass [J]. Handicraft teaching, 2013, (4): 100-102.

[3] Shi Quan. A practical hand shaped glass edge grinding method [J]. Glass, 2012, 39 (7): 36-37.

[4] Tao Weiqing, Adachi m. Glass laser engraving machine control system design [J]. Micro computer information: automatic measure and control instrument, 2002, (7): 28-29.

[5] Liu Qihong. CAD / CAM CNC glass engraving system[J].Micro computer information: instrument automation, 2003, (1): 28-29. 